HStud 28 (2014)2, 315-332

DOI: 10.1556/HStud.28.2014.2.7

\title{
FROM CABERNET SAUVIGNON TO EGRI CSILLAG: CHANGING PATTERNS IN HUNGARIAN WINE NAMING
}

\author{
ERZSÉBET TÓTH-CZIFRA \\ Eötvös Loránd University, Budapest, Hungary \\ E-mail: toth.czifrae@gmail.com
}

\begin{abstract}
This paper aims to demonstrate the changing practice of Hungarian wine branding and wine naming. I show how the branding and naming strategies react to the recent changes in the field of wine selling and consumption in Hungary. These changes increased the importance of the front label in wine selling and, as a result, the number of creative wine names increased significantly. I adopt a combined approach of corpus and cognitive linguistics and make the following hypothesis: due to the complex function of brand and product names - i.e. to identify the product, and to catch consumers' attention and therefore help in imprinting - branding and naming strategies are governed by the minimax principle (Berkle 1978). By providing a cognitive corpus linguistic analysis of a collection of wine names, I aim to identify newly emergent naming schemata. In doing so, I demonstrate that the increasing richness of the novel brand and product names is a result of a set of conceptual mechanisms underlying their semantic make up (Hernandez-Pérez 2013). These are: metonymy, metaphor, conceptual integration and phonological analogy.
\end{abstract}

Keywords: viticulture, marketing, cognitive linguistics, branding, naming strategies, Hungarian wine

\section{Introduction - On the Increasing Importance of the Front Label}

In the first 15 years following the 1989 change of regimes in Hungary, significant changes occurred in the structure of wine production as the cc. 40 wine-producing cooperatives ceded their places to an increasing number of small, mostly family owned wineries (Farkas 2007). Similar to the international trends (see Walker 2006, Lehrer 2009: 239 and 2013: 12), the hypermarkets and large wine shops caused changes in wine selling and, as a result, in wine naming. These recent developments, accompanied by the development of wine tourism and festivals, brought a wider selection and an increasingly competitive environment. ${ }^{1}$ The surface of the bottle, which originally had the primary function of identifying the product by providing information on the characteristics of the wine, became an important place for advertisement (in fact, for a large portion of Hungarian wines 
the only one). One of the most important tools of designing an eye-catching label that attracts consumers' attention is to increase the suggestiveness and semantic richness of the product names. ${ }^{2}$

One could argue that today's marketing objectives demand double functionality from a wine name. Primarily, it should be congruent with the target product. That is, both brand name and product name have to provide a unique reference in order to identify and position the product within its category. Secondly, the name has to gain a selling power that is capable of catching the attention of the consumers and can prompt inferences based on the rich semantic fabric encoded in the product name. The question is, how these two different functions can be fulfilled by a given name. To answer, we have to take an excursion to the meaningfulness of these names.

Concerning the international presence of creative wine names, Adrianne Lehrer addresses the question as to whether these names are meaningful. According to her, " (...) 'technically' they do not have meaning, but they take on the associations connected to the word or words selected (Lehrer 1992)" (Lehrer 2013: 9-10). Contrary to this approach, I consider wine names as being systematically motivated by a certain set of cognitive principles, whose creativity cannot originate from purely fictitious sources since they have to achieve relevance to their target product and fulfill the aforementioned identifying function. Accordingly, it can be observed how the two afore mentioned functions mutually constrain each other: the eye-catching properties have to be congruent with the identification and positioning of the product, and the other way round. In order to understand how these two emerge in a successful product name we have to take minimax principle into consideration (Berkle 1978) which is mainly used in the investigation of compounds (see Benczes 2010). According to this principle, the economy of compounds reflects a minimum of surface complexity combined with a maximum of semantic information expressible. The application of this principle within the semantic field of wine names concerns 1) the amount of the information that provides a unique reference for the given wine and helps categorize and position the product on the market and 2) the maximization of the possible evocative content, i.e. positive connotations and aspects of meaning that increase the selling power of the name.

\section{Hypotheses}

Based on the above, I hypothesize that 1 . Hungarian wine names today are meaningful symbolic units which have a double function; 2. A higher degree of cognitive economy is needed in order to fulfill both of these functions. This economy, reflected in the minimax principle, shapes the semantic configuration of wine 
names; 3 . As a result, the semantic richness of these names is carried out by meaning extensions of different kinds which convey a wealth of meaning that goes beyond the literal interpretation of the chosen name. Therefore wine names are figurative in nature. Before analyzing each of them in relation to the Hungarian wine names, I introduce the corpora on which the research was based.

Table 1. Distributions of the corpora of brand and product names

\begin{tabular}{lccc}
\hline Source & Period & $\begin{array}{c}\text { Number of } \\
\text { product names }\end{array}$ & $\begin{array}{c}\text { Number of } \\
\text { brand names }\end{array}$ \\
\hline Bortársaság & 2014 & 421 & 80 \\
Annual selection & 2006 and 2008 & 200 & 78 \\
\hline In total & $2014,2006,2008$ & 621 & 114 \\
\hline
\end{tabular}

\section{The Examined Corpora}

In order to be representative of today's wine naming practices, the basis of the corpus was obtained by listing all the items from Hungary's biggest wine distributor company, the Bortársaság's web page (Hungarian Wine Society, www.bortarsasag.hu), which provides a good cross-section overview of the Hungarian brand and product names available on the market. ${ }^{3}$ This means 421 product names from 80 brands. In order to account for the patterns in their changes over a short period, this collection was complemented by another corpus containing 200 product names and 78 brand names respectively ${ }^{4}$ from the years 2006 and $2008 . .^{5}$ In total, a corpus of 621 product names and 114 brand names ${ }^{6}$ provides the basis of the present investigation. In the followings, I will analyze this collection by looking into the pragmatic and semantic mechanisms underlying their semantic configuration and touch upon the differences in the naming strategies characteristic of the two periods.

\section{Branding}

Regarding the branding strategies, the picture is rather homogenous. The metonymy PRODUCER FOR PRODUCT is by far the most productive mechanism underlying Hungarian wine brands $(87 \%$ of the corpus instantiated this metonymy). Since even the most creative and novel wine names retain this traditional way of branding, the question arises, what the origins of the strength of this conceptual link are, and why this pattern is so dominant. 
First of all, family names, as proper nouns, function as "rigid designators of a unique reference" (Barcelona 2003: 12). The (family) name of the wine producer is not only more salient than any other element of the WINERY frame such as the terroire, the wine yard or the grape varietal the wine is made of, but is also capable of identifying the product in a straightforward way. Therefore, the uniquely identifying function of brand names is fulfilled. Another reason for the family name being preferred over other nouns (e.g. region, winery) is the fact of the highly fragmented ownership of the wine regions. While in other countries a region is suitable to identify the product (e.g. Chianti, Bordeaux), in Hungary these links are too general and therefore do not provide differentia specifica. Now let us see in which ways this naming schema is capable of giving rise to positive connotations. The following analysis is based on the theoretical framework of Cognitive Metaphor Theory (Lakoff and Johnson 1980).

One important implication of the PRODUCER FOR PRODUCT metonymy is that its source domain also implies a sense of guarantee in the consumer inasmuch as the winemaker gives his good name (and sometimes even his face or portrait) to the product, communicating personal responsibility. In some cases this metonymy is further elaborated by the product name (see e.g. Gere Attila Cuvée 2007; Thummerer Vili papa Cuvée 2006 ['Thummerer Uncle Vili Cuvée']). These advantageous attributes are also strengthened by the fact that the producer for product metonymy as branding schema is usually preferred in the domain of luxury goods (e.g. Ray Ban, Gucci). This, as a priming effect, might also contribute to the development of positive connotations. Notice, however, that the wealth of knowledge that becomes activated within the winery frame this way heavily depends on whether and to what extent buyers are "wineologically skilled" (Lehrer 2009) or familiar with the Hungarian wine-producing context. Knowledge of winemaker generations or recognition of the producer as the founder of the company (which is often the case in Hungary) has a potential to enrich the semantics of the brand by activating a whole metonymic chain ranging from the founder through the wine producer accounting for the target product itself. Although all this information is not necessarily available for consumers, the family business nature of the winery is clearly indicated by the source, even for those who are less familiar with the Hungarian wine-producing context. Wine brands therefore reflect sensitively the type of business enterprise and therefore convey the sense of added values, like family tradition and craftsmanship. This accurate reflection of production and ownership in wine names is even more apparent if we consider that in those cases where - as a deviation from the conventional Hungarian wine branding schema names make use of conceptual mechanisms other than that of the producer for product metonymy (e.g. IKON, Folly Arboretum, Pannonhalmi Apátság), the owner of the winery is not a family but an institution or corporation. 
One potential reason for the homogenous and rather traditional branding practice in the case of Hungarian wine names in particular and wines in general is the partial distribution among the functions of brand names and product names. From their general functions identified in the previous section, brand names serve as the primary identifying cues, therefore product names have more room to be playful and more descriptive in this space already marked by the brand name.

\section{The Traditional Pattern of Wine Naming}

Taking one step closer to the content of the bottle, in the following, I will focus on the subordinate category of branding, namely, the actual product names. As for Hungarian wine names, the ones that instantiate the traditional pattern also stay within the WINERY frame and take one of its subdomain, most frequently the grape varietal $^{7}$ the wine is made of (Cabernet Sauvignon, Furmint, etc.), the place of production (Kopár) or simply the color of the wine (Red, White), or, in addition, the actual technique used (e.g barrique, late harvest) to provide access to the target, the wine. These names, on the one hand, further specify the characteristics of the wine and are rather descriptive and straightforwardly informative. As an example, the wine name in (1) is a prototypical instantiation of this metonymic pattern. The brand designates a family name, Merlot is the grape varietal, while Barrique refers to the technique applied in the winemaking process.

\section{(1) Günzer Merlot Barrique 2008}

The cognitive mechanism that gives rise to this naming schema is again a metonymy, where THE GRAPE VARIETAL THE WINE IS MADE OF STANDS FOR THE PRODUCT. The conceptual content metonymies like this activate is strongly consistent with the target product and therefore achieve strong relevance with it. By foregrounding one (or two) of the salient characteristics of the wines, conventional metonymic names complete the task of categorizing and therefore make products identifiable among wines in general and within the brand in particular. Furthermore, one important advantage of making use of the grape varietal the wine is made of is making the type of wine internationally recognizable. ${ }^{8}$ At the same time, however this naming schema assumes that consumers are familiar with grape varietals.

Looking at the corpora, it seems that in spite of its decreasing proportion, this naming pattern is still the most frequent one. As the data shows, $91 \%$ of the examples in the 2006/2008 corpus fall into this pattern, while in the 2014 corpus this proportion had decreased to $79 \%$. This decrease is particularly significant, especially if we consider that there is only a 5 -year period between the two corpora. 


\section{Creative Wine Naming}

This group consists of the wine names which deviate from the above depicted traditional pattern. Common characteristics of them are: 1. a set of cognitive operations, metonymy is only one of them, underlying their semantic configuration. These are: metaphor, metonymy, conceptual integration, complemented by analogy and rhyming. 2. In order to maximize the evocative effect of the name in target in many cases more than one of them are at work. 3. Some of these names work at multiple levels inasmuch as their effect is largely influenced by consumers' approach to and knowledge of context (i.e. interpreting from the perspective of a wider cultural context or from the context of professional winespeak with all its lexical and discursive knowledge).

\section{Metonymy-based Creative Wine Names}

Metonymy-based creative names are the closest relatives of the traditional pattern, since in many cases only a slight modification distinguishes them from the names representing the traditional naming pattern. For instance, in Légli Lugas there is an unconventional instantiation of the traditional PLACE OF PRODUCTION FOR THE PRODUCT (MADE THERE) pattern. Instead of making use of the relevant wineyard of wine producing area, a common noun, designating an informal wine-related place, is chosen for source domain. As lugas (trellis) is seen as a cozy place for wine consumption as well, wealth of its positive connotations are also mapped onto the name. In addition, the sound symbolic effect ${ }^{9}$ of the name also contributes to the relaxed atmosphere evoked by the source. Thus, the congruence of the semantic and phonetic pole of the name therefore increases the semantic efficiency of the resulting product name

In one form of metonymy-based creative names the WINEMAKER (or, the WINEMAKER FAMILY) subdomain of the WINERY ICM activated by the brand is further elaborated in the product name and therefore strengthen all its positive effects (i.e. the personal guarantee, the familiarity, etc. see above).

\section{(2) a. Gere Attila Cuvée 2007 \\ b. Thummerer Vili papa Cuvée 2006 \\ ('Thummerer Uncle Vili Cuvée')}

Comparing (2) a. and b. it can be seen that their different connotations are carried out by the lexical choice. In the case of (2) a. Gere is the brand name and Attila Cuvée is the product name. Together (Gere Attila Cuvée) explicits the name of the founder and head of the winery ${ }^{10}$ (and of the family as well) and therefore refer to the high-end status of the actual wine (cf. LEADER OF THE WINERY FOR THE 
LEADING PRODUCT). The name in handwriting (see Fig. 1) helps in interpreting the product as an ars-poetica even for those not familiar with the Hungarian winemakers in general. The example in (2) b. bears totally different connotations with evoking a familiar atmosphere by explicating the family member status of the winemaker.

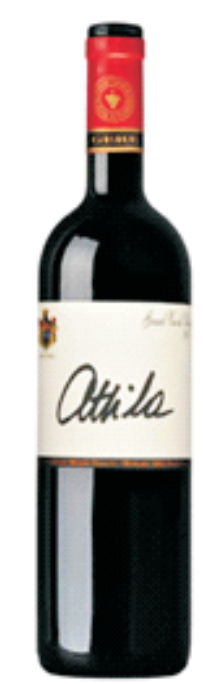

Figure 1. Handwriting stands for the personal guarantee on the bottle of Gere Attila Cuvée

Another type of productive metonymical source domains is that of the effects and reactions triggered by the consumption of a particular wine. Látomás ('vision') and Boldogságos ('happy') are good examples instantiating this CAUSE FOR EFFECT mapping, since both are positive effects and are made to stand for the wine that causes them, thus presenting the target product as something desirable. The name Aligvárom ('Icantwait') reflects straightforwardly the desirableness of the given wine as the FEELINGS TOWARDS THE PRODUCT STAND FOR THE PRODUCT. Furthermore, the unusual way of nominalization (a whole phrase is nominalized) also adds to the salience of the name. The persuasive power of these metonymies originates from the hyperbolic way in which the products' positive effects are conceptualized. Also, these names clearly illustrate how the purpose of the communicative exchange (i.e. selling wine) governs the selection of the elements from the effect frame (e.g. although 'hangover', as an effect, is highly relevant for wines, due to its negative connotations its selection for being mapped into a name is straightforwardly ruled out). 
In the third type of metonymy-based creative wine names we find PART FOR WHOLE mappings in that one of the subdomains of the product ICM stands for the product itself. A careful selection of the most relevant, desirable or prolific characteristics, like in Friss ('fresh') or Illatos Friss ('fragrant fresh') increases the efficiency of the resulting name. The lexical choice of the selected attribute can also contribute to the intended meaning and deepen the conceptual link between the name and its referent. Consider Szekszárdi Ó Vörös ('old red from Szekszárd') where an old word meaning old is chosen to refer to a wine whose quality increases with age.

\section{Making Use of Knowledge on the Self and on the Other in Metonymy-based Wine Names}

Hungarian wine names of this type have a preference for describing the product with terms generally used in the context of folk and fairy tales. Mesés ('fairy','faboulous', 'starry'), Madárlátta (lit. 'seen by the birds', expression for something travelled around), Százrejtekü ('hundredfold'), Hetedhét vörös ('once upon a red') ${ }^{11}$ do not only refer to highly culture-specific concepts which are difficult to translate, but as such, also add positive connotations from the FOLK TALE frame. Additionally, through these expressions the product is placed into a specific scenario or a narrative typical of tales and deeply entrenched into the Hungarian culture.

Interestingly, though in lower numbers, the same conceptual mechanism (i.e. metonymically foregrounding a positive attribute of the product instantiating the SALIENT PROPERTY FOR THE PRODUCT metonymy) is present with source concepts, which implies the sense of otherness instead of culturally deeply entrenched concepts. Product names like Fortissimo and Andante activate the highly sophisticated atmosphere of arts, since these are used for the characterization of classical music. Moreover, this naming pattern is congruent with the brand name concerned (Gróf Degenfeld, 'Earl of Degenfeld') which also suggests nobility and exclusiveness. It depends on consumers' attitude towards classical music in particular or Italian language in general whether connotations like elegance, exclusiveness and nobility will be inferred or, as an opposite effect, they interpret the name as a sign of snobbism or expensive price.

In this section I demonstrated how metonymy results in meaning extensions in the field of Hungarian wine names. All the sources produce different communicative effects which has several advantages in designing an effective and eye-catching product name. Staying within either the WINERY or the WINE frames results in straightforward consistency with the product through mapping felicitous descriptive information into the product names. Their promotional aspect is carried out 
via carefully chosen source concepts, often hyperbolic in nature, as well as via lexical choice which further elaborates the conceptual basis of them. One important consequence of deviation from the traditional naming schema is that it enables the products to address wider audience. Choosing a more general, culturally entrenched adjective as a source concept for this purpose - instead the grape varietal the wine is made of - activates a wider and within Hungarian culture generally shared context rather than that of wine knowledge. As a result, these creative names become accessible also for those who have no idea what type of wine a Pinot Noir is. At the same time, however, this makes them inaccessible for the international audience. Considering the fact that the wines in question are meant to be sold in Hungary, it can be stated that their names reflect and show high degree of consistency with their target audience.

\section{Metaphor-based Creative Wine Names}

Involving across-domain mappings, the greater conceptual distance between the two independent domains (source domain and target domain) makes metaphor a flexible tool for naming. In this naming pattern the descriptive content of names is no more readily accessible and therefore more emphasis falls on the process of interpretation. One aspect of the conformity to the minimax principle is that metaphorical wine names are interpretable on multiple levels, according to what extent the potential consumer is familiar with the professional context of wine drinking or wine speak. Therefore these names can be interpreted inside the already existing wine discourse metaphors, as elaborations of them, or outside, but not independently from the characteristics of the wine. As a result of this type of cognitive operation a large number of resemblance metaphors (Kövecses 2013) were found in the corpus. According to the matrix of wine metaphors developed in Hernandez-Pérez (2013), we can distinguish them on the basis of whether wine is compared to inanimate objects or living entities. This latter group proved to be preferred over the former, as, according to the data, Hungarian wine brands largely exploit the general-level metaphor NON-LIVING ENTITIES ARE LIVING ENTITIES which yields two specific-level metaphors: WINES ARE ANIMALS and WINES ARE PEOPLE.

The name Tinta ('ink') illustrates how an inanimate object is capable of extending the meaning of the target (INANIMATE OBJECTS ARE WINES) and identifying its type at the same time. Both the color and the liquid form of the source (and also the circumstance that ink is stored in a dark bottle) are mapped onto the target, therefore, one can conceptualize the given bottle of wine as if a bottle of ink. In the hyperbolic effect of the color mapping, namely, that the wine does not, actually, reach such an intense tonality that ink does, we can observe the operation of what 
is called strengthening (Hernandez-Pérez 2011: 44). Strengthening proved to be widespread in the practice of Hungarian wine naming (see also Elixir, Indigó, Essenzia, etc.). In addition, the name Tinta also makes use of the Hungarian slang expression betintázik (lit. 'inking in') which refers to getting drunk or tipsy but tends to have a more permissive and funny evaluation. This way the name achieves congruency with the context of wine consumption through a sense of wittiness.

One important attribute of conceptual metaphors which enables metaphorical wine names to fulfil the communicative need for being persuasive is that each conceptual metaphor imposes its own particular perspective through the metaphorical linguistic expressions chosen (Lakoff 1996). Accordingly, metaphorical product names not only describe but also evaluate the product. Wine names making use of the INANIMATE OBJECTS ARE LIVING ENTITIES general-level metaphor may be particularly persuasive because of two reasons. First, living entities have greater cognitive salience than non-living entities (Radden and Kövecses 1999). Second, and in connection with the former, these source domains imply a shift in the GREAT CHAIN OF BEING metaphor and position them as a higher form of existence in this culturally embedded hierarchy of entities. Furthermore, personification is a powerful branding tool in general (see Kövecses 2010).

As instantiations of the INANIMATE OBJECTS ARE PEOPLE, more specifically, wines are people metaphor, source domains, like Barbár ('barbarian') and Nomád ('nomad') are interpretable as further elaborations of the already existing metaphors from the wine discourse. The (stereo)typical traits of a barbarian person are strength, wildness and roughness. All these adjectives are conventionally used construals in wine speak regarding either the body or the astringency (see Lehrer 2009) of a red wine. ${ }^{12}$ This example also shows how the gender aspect of the wines are people metaphor is entrenched in the already existing ways of conceptualizing and talking about wines, i.e. in wine speak red wines tend to be treated as masculine, while white wines are rather conceptualized as feminine beings. ${ }^{13}$ Notice, however, that in Hungarian culture (and, presumably, in many others) barbarian carries mainly negative connotations. Though wildness and roughness in the wine speak are considered as positive characteristics often describing a high-positioned red wine, outside of this context, i.e. in the generally shared Hungarian (or even wider) cultural context barbarians are rather synonyms of unculturedness and uneducatedness. Accordingly, in the case of a wine called Barbár the degree of familiarity with the wine discourse facilitates the effectiveness of the wine name, i.e. whether its positive connotations originating from the wine discourse are foregrounded in the interpretation, or the more generally accessible negative ones.

Similarly to the international wine naming practice (see Walker 2006), animals are frequently used sources also among Hungarian wine names. Basically three 
naming schemata can be observed in the corpora which make use of the WINES ARE ANIMALS metaphor. With regard to the first, Oroszlán ('lion') is a good example, which simply maps the characteristics of the animal onto the wine. As it was touched upon above in the introduction, the selection of the relevant attributes of the stereotypical (or: collectively shared) mental representation of a lion is governed by the contextual factor called topic of the discourse (i.e. the target product) (see Fig. 2). Apart from attention raising and providing an easy-to-memorize cue to the consumer this naming strategy also involves the positioning of the product within the brand hierarchy. ${ }^{14}$

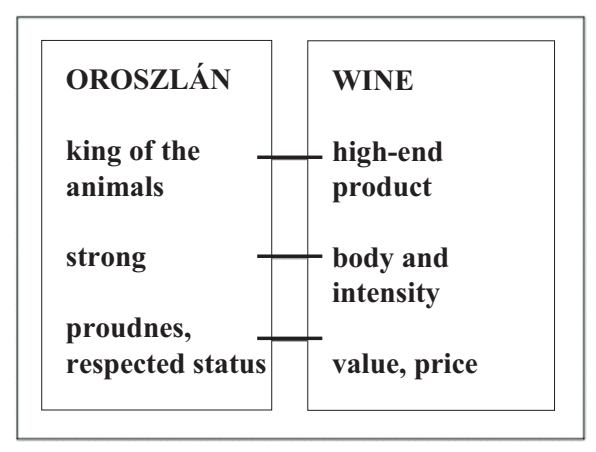

Figure 2. The metaphoric mappings of a wine conceptualized as a lion

Applying this pattern to another instance of the animal product names we find that in the case of Farkas Furmint or Medve Furmint the traits one can associate from these animals are not congruent with the attributes of the wine in question. Taking a closer look at the motivation underlying this second naming schema, it turns out that these names rather have a metonymic basis, since the animals are taken from the name of the wine yard (Farkas dülö, Medve dülö) where the wine was produced and therefore represent the given geographic area and refer to historical fauna rather than to the wine. Since this element of the WINERY frame is not a widely shared one, the accessibility of this semantic motivation is strongly limited (that is, only for those who have detailed knowledge on local (historical) myths and beliefs). Thus, a name which achieves consistency with the environment where wine is produced instead of the wine itself, and therefore provides no descriptive cues to help in the identification of the product, can be considered as a misleading one with reduced efficiency.

The optimal solution is a third naming schema involving animals where the above described patterns are merged and the multiple contextual cues activated result in and achieve a higher degree of relevance. In the corpora examples can be found for this third pattern as well. For instance, the name Ördög ('devil') both re- 
fers to the origin of the wine (Ördögárok dülö, which is a wine yard with high-quality terrain) and at the same time makes use of some advantageous characteristics of the shared mental representation of 'ördög' which are relevant for and mapped onto the target product. Since this example has the capability of expanding the metaphorical naming schema, before I touch upon the semantic configuration of the naming of this type, a brief summary is provided on what the analysis revealed on metaphoric wine names.

As can be seen, metaphor-based wine names reflect a higher degree of deviation from the traditional Hungarian naming schema, since making inferences between two independent and conceptually distant domains (such as that of ANIMALS, PEOPLE or OBJECTS like INK and WINE) requires greater cognitive effort and therefore more emphasis falls on the process of interpretation. This makes metaphor a flexible tool for naming where the descriptive content of names is no more readily accessible but inferred. As a result, metaphorical wine names are interpretable on multiple levels due to consumers' access to contextual factors of different sorts. Here persuasiveness was achieved either through the (pragmatic) strategy of strengthening, or by procuring a shift in the GREAT CHAIN OF BEINGS metaphor which has the advantage of positioning the target product into a higher order of existence. Both of these strategies manifest in the careful selection of the source material.

\section{Conceptual Integration in Creative Wine Names}

Making use of both the origin of the wine and some of the characteristics of $\ddot{o r d o g}$ ('devil') implies that in order to fully exploit the semantic richness of the name hearers have to simultaneously activate two different frames: WINERY frame (i.e. the quality of the grape grown in Ördögárok dülö) and another one from our culturally shared mental representation of ÖRDÖG. The interpretation of this semantic complexity can be accounted for neither with the purely metaphoric, nor with the purely metonymic reading. ${ }^{15}$ In the following, therefore, I will analyze the wine name $\ddot{O r d o ̈ g}$ as a double scope conceptual integration ${ }^{16}$ where the two input spaces are the winery frame and the ördög (devil) frame, while the blended space is the wine itself. This interpretation is illustrated by Figure 3 where the metonymic mappings are indicated with dashed lines, while the metaphoric ones are indicated with bold lines. In this interpretation the culture-specific conceptualization of the devil (ördög) deserves a mention.

In the Hungarian concept the supernatural power and skillfulness of devil is foregrounded and - apart from its real "devilness" - results in positive connotations. This is reflected in idiomatic expressions like ördögien jó ('devilishly good') or az ördög hegedüse ('violinist of the Devil', whose referent is conven- 


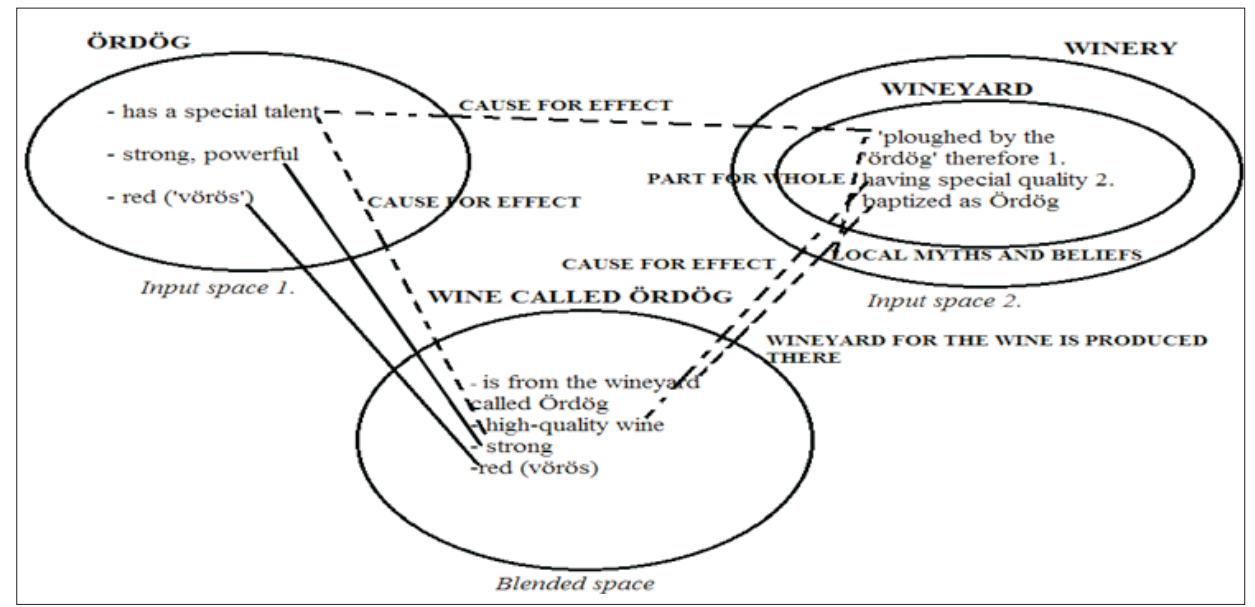

Figure 3. Possible interpretation of the wine Vylyan Ördög

tionally the famous $19^{\text {th }}$ century violin virtuoso Niccoló Paganini). Another relevant aspect of this within-culture shared mental representation of 'ördög' is its red color. ${ }^{17}$ Furthermore, here the same preference for the FOLK TALES frame is present, previously witnessed in a group of metonymic names (e.g. Mesés, Százrejtekü, Hetedhét vörös).

The semantic complexity of conceptual integration-based names leads us to the cognitive operation exploiting the minimax principle to the full. It depends on the knowledge of the context whether these are interpreted as pure metonymies, pure metaphors or both.

One of the most obvious differences in the 2013 corpus in comparison to the 2006-08 one is the increasing number of names based on multiple conceptual operations i.e. names that have the potential for instantiating conceptual integration. ${ }^{18}$ In the 2014 corpus I found 16 of them while in the 2006/2008 corpus only one. Names making use of conceptual integration are good candidates for product names because they are capable of making extensive use of the minimax principle in that the complex network of conceptual mappings at work in a conceptual integration is able to achieve a maximum semantic effect and information with a minimum of effort. According to Benczes (2010) this maximum effect might also involve humor.

A general characteristic of these examples is that they rely on highly culture-specific concepts and linguistic units and therefore some of them are impossible to translate. In the following example it is demonstrated how making use of an elementary piece of cultural knowledge can trigger an efficient product name with a high degree of relevance. Egri Csillag ('Star from Eger') is created through a reduction of a proper name, Géza Gárdonyi's novel, Egri Csillagok (Stars from 
Eger). This title is deeply entrenched in Hungarian common cultural knowledge, practically known by every Hungarian, since it is one of the earliest piece of the Hungarian literary canon taught in elementary schools. As such, it is a salient source referring to a product of the same origin (see Fig. 4). The reduction (and also the possible remotivation) of the proper name involves the metaphoric mapping THE WINE IS A STAR OF EGER, and therefore places the product into an honorable historical narrative and a cultural context. Furthermore, the star accounts for the high value of the wine as well.

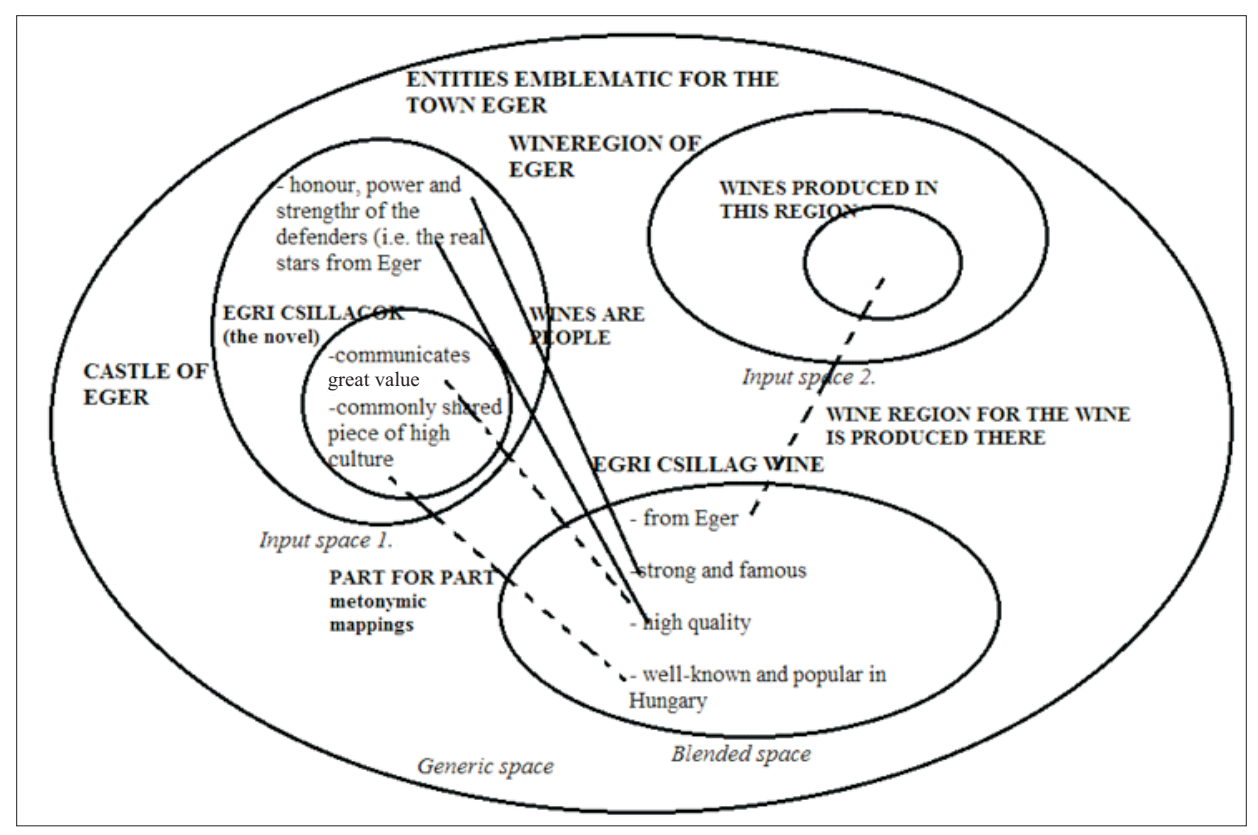

Figure 4. Possible interpretation of the wine Egri Csillag

As it can be seen in Figure 4 here the umbrella term ENTITIES EMBLEMATIC FOR EGER provides the generic space of the conceptual integration. This represents conventional cultural knowledge attached to the town. Two especially relevant and therefore salient pieces of it serve as input spaces, namely, WINE REGION OF EGER and CASTLE OF EGER. The complex network of metaphoric (bold lines) and metonymic (dashed lines) mappings result in the rich semantic configuration of the blended space i.e. the wine name Egri Csillag.

Apart from making use of within-culture deeply entrenched concepts, in another portion of conceptual integration-based names the opposite tendency is also present, that is, preference for source concepts which implies the sense of otherness. Here, either an English term is used (e.g. Magic Rain), and therefore can be interpreted cross-culturally, or the name interacts with foreign languages and cul- 
tures reflecting some analogy or linguistic hybridity. Pholonogical analogy-based conceptual integrations usually involve puns in the product names such as: Porta Géza which rhymes to portugieser, Mammaróza Rozé which rhymes with rosé, and Bogyólé (lit. 'berry juice') which evokes Beaujolais.

This latter represents a higher degree of semantic complexity. Bogyólé ('berry juice') is a metaphor-based name which is congruent with wine speak in that comparing the aroma and bouquet of (good) wines to berries of different kinds is a usual aspect of wine descriptions (cf. the recontextualization of the sensory experience of wine drinking). The source concept berry juice therefore highlights a salient and positive characteristic of the wine, namely, its intensive and pleasant aroma and bouquet. Thanks to the phonological similarity, when coming across Bogyólé the hearer (consumer) can also access Beaujolais, which considerably contributes to the semantic enrichment of the name and also helps to unravel the meaning of it. Additionally, Beaujolais refers not only to a special early harvest type of wine and the special wine producing technique, but also to the town famous for its production (cf. the paragon use of the place name), therefore, the name makes use of the double metonymy PLACE FOR THE TYPE OF WINE TYPICALLY PRODUCED THERE AND GENERAL TYPE OF WINE FOR THE SPECIFIC WINE. Bogyólé thus can be interpreted as a "Hungarianized version" of Beaujolais (at least linguistically). These two source concepts achieve a high degree of congruency with each other since one general characteristic of the Beaujolais types of wines is their rich berry-like overtones in their aroma. Therefore, phonological analogy to an already existing compound in this case is able to bring forth conceptual similarities between the source name and the novel coinage, thereby enhancing the humorous effect of the novel expression.

In this section the latest and most creative Hungarian wine naming practice was described. The resulting product names motivated by multiple cognitive operations i.e. their (complex) semantic configuration is both metaphor- and metonymy-based, and as such, were analyzed as conceptual integrations. These names are capable of exploiting the minimax principle in that the complex network of conceptual mappings at work in a conceptual integration is able to achieve a maximum semantic effect and information with a minimum surface complexity. As it has been demonstrated, this maximum effect might also involve humor.

\section{Concluding Remarks}

The aim of this paper was to investigate how the recent changes in wine market and consumption affected Hungarian wine branding and naming. The corpus-based cognitive analysis of Hungarian wine names in the years 2006 and 2008, and 2014, respectively, has revealed a tendency of shift from the traditional 
naming schema towards unconventional, creative names. As a result, new naming schemas with different pragmatic and semantic mechanisms underlying their semantic configuration have emerged. The semantic richness of these novel brand and product naming patterns is a result of a set of conceptual mechanisms underlying them systematically. These are: metonymy, metaphor, and conceptual integration complemented by phonological analogy.

In the naming strategies embodied in naming schemas the multiple functions an effective name has to fulfil are present at different levels and degrees. That is, in branding and traditional naming strategies the straightforward (and conventional) categorization and identification is more dominant than the attention-seeking or descriptive character. In some of them making use of proper names (like family names, names of wine producers or place names) guarantees a unique reference to the target product. Due to their conventionality, their interpretation requires familiarity and knowledge on wines and wine discourse. In the case of recently emerged creative wine names on the contrary, due to their unconventional nature, multiple context-sensitivity and higher degree of semantic complexity, the (figurative) inference-making process itself is foregrounded, which makes the resulting creative names more flexible for interpretation. The rich and often humorous and witty imagery encoded in them with the use of conceptual operations of different sorts makes them attractive and attention-grabbing. These qualities enable wine names to fulfil their complex functions.

A general characteristic of these strategies is that in them often highly culture-specific concepts are made use of and as such, it reflects both the origin and the target audience of the products.

Furthermore, the cognitive analysis of Hungarian wine names suggests that semantic complexity goes together with increasing flexibility regarding their interpretation. The fact that these resulting creative names are open for multiple interpretations from different perspectives presumably enables product names to be accessible for a wide and heterogeneous audience. This hypothesis is only one of the many emerged during the analysis. As a possible continuation of the research, a detailed consumer preference survey could shed light on the in-practice functioning of the naming schemas identified in this paper and provide an in-depth insight on the validity of these hypotheses. 


\section{References}

Barcelona, A. 2003. Names: A Metonymic 'Return Ticket' in Five Languages. Jezikoslovlje 4(1), $11-41$.

Benczes, R. 2010. Setting Limits on Creativity in the Production and Use of Metaphorical and Metonymical Compounds. In: Sascha, Michel and Onysko, Alexander (eds): Cognitive Approaches to Word Formation. Berlin and New York: Mouton de Gruyter, 221-45.

Benczes, R. 2013. The Role of Alliteration and Rhyme in Novel Metaphorical and Metonymical Compounds. Metaphor and Symbol 18(3), 167-84.

Benczes, R. and Tóth-Czifra, E. 2014. The Hungarian Colour Terms piros and vörös. A Corpus and Cognitive Linguistic Account. Acta Linguistica Hungarica 61(2), 123-52.

Farkas, Z. 2007. Hungary: Brand New World of Traditional Winemaking. Wine Business International 2007(12). Web. [Accessed 23 June 2014.]

Fauconnier, G. and Turner, M. 2002. The Way We Think: Conceptual Blending \& the Mind's Hidden Complexities. New York: Basic Books.

Hernández-Pérez, L. P. 2013. A Pragmatic-Cognitive Approach to Brand Names: A Case Study of Rioja Wine Brands. Names: A Journal of Onomastics 61(1), 33-46.

Issekutz, E. (ed.) 2008. A száz legjobb magyar bor [Top 100 Hungarian Wines]. Budapest: Népszabadság.

Kövecses, Z. 2002. Metaphor: A Practical Introduction. Oxford and New York: Oxford University Press.

Kövecses, Z. 2006. Language, Mind, and Culture. A Practical Introduction. New York: Oxford University Press.

Kövecses, Z. 2010. Metaphor: A Practical Introduction. Second edition. Oxford and New York: Oxford University Press.

Kövecses, Z. 2013. The Metaphor-Metonymy Relationship: Correlation Metaphors are Based on Metonymy. Metaphor and Symbol 28(2), 75-88.

László, Á. and Issekutz, E. (eds) 2006. A száz legjobb magyar bor. Budapest: Népszabadság.

Lakoff, G. and Johnson, M. 1980. Metaphors We Live by. Chicago: The University of Chicago Press.

Lehrer, A. 2009. Wine and Conversation. Oxford University Press.

Lehrer, A. 2013. Trendy New Trends in Wine Terminology. Unpublished manuscript.

Paradis, C. and Olofsson, M. E. 2013. Describing Sensory Experience: The Genre of Wine Reviews. Metaphor \& Symbol 28(1), 22-40.

Radden, G. and Kövecses, Z. 1999. Towards a Theory of Metonymy. In: Panther, K.-U. and Radden, G. (eds), Metonymy in Language and Thought. Amsterdam - Philadelphia, 17-59.

Yorkston, E. A. and Menon, G. 2004. A Sound Idea: Phonetic Effects of Brand Names on Consumer Judgments. Journal of Consumer Research 31/2008, 43-51.

\section{Notes}

1 For more information on Hungarian wine producing, see Farkas (2007).

2 Certainly, the textual part of the label is not the only effective cognitive tool for a successful branding. A wide range of visual elements and perhaps even some degree of tactile experience can and do interact with the names. Although these interactions might form a basis for a multimodal cognitive research, these other modalities are out of the scope of this paper.

3 This corpus was designed in June 2014. 
4 The source of this collection is the annual selection: A száz legjobb magyar bor [Top 100 Hungarian Wines]. (László and Issekutz 2006; Issekutz 2008.)

5 In the year 2007 this collection was not published.

6 Due to overlapping, the brand names in the two corpora are not added up. This is not the case regarding the product names where at least the year differentiates the otherwise similar names.

7 Interesting that some of these are already figurative in nature, see e.g. királyleányka ('princessin') or ezerjó ('thousands of good'). Since these are well entrenched symbolic units, their figurative nature is not active during the course of interpretation. As highly conventionalized symbolic units, despite their internal complexity, these units "constitute for the speaker "prepackaged" assemblies because he has no need to reflect on how to put it together" (Langacker 1987: 57).

8 As the provisions of the (Hungarian) Act XVIII of 2004 on grape and wine producing state, the Hungarian producers have to indicate the grape varietal according to international naming standards.

9 On how sound symbolic effect can and does influence consumers' choice see Yorkston and Menon (2004).

10 Since this alignment is congruent with the Hungarian naming practice (i.e. family name fist and then the given name), this wine name further elaborates the signature-like character of the wine.

11 For the creative and sensitive translations I wish to thank Judit Pethö-Szirmai.

12 Interestingly and surprisingly enough, no research has been carried out on Hungarian wine discourse yet. Therefore, in the following I have to rely on 1. the English language wine speak, 2. wine descriptions available on the homepage of Bortársaság, 3. my own introspection regarding Hungarian wine discourse.

13 Although as Lehrer (2013:3) points out this practice is fading in western culture because of its sexist overtones.

14 Not surprisingly the data shows that the naming of the high-end products usually receives more attention and creative effort.

15 Nevertheless, the interpretation of these names either as a pure metaphor or as a pure metonymy is also possible depending on the knowledge accessible for the consumers. E.g. when a potential consumer is not familiar with the fact that the target product is made from grape grown in the Ördögárok wineyard, the metaphorical interpretation (wine is a devil) might come into play. This example illustrates the fuzzy boundaries between instances of conceptual metaphor and conceptual integration.

16 Here I adopt the Fauconnier-Turner (2002) view of conceptual integration. Conceptual integration occurs "when mental spaces do not simply map onto each other but partially blend their conceptual content" (Kövecses 2006). Conceptual integration (or a conceptual blend) consists of input spaces (the one with one input space is called a single scope blend while the one with two input spaces is a double scope blend), a blended space and (not necessarily) a generic space, a frame within which the whole conceptual operation is interpretable.

17 An interesting aspect of the Hungarian system of color terms is that as the equvivalent of the English 'red' in Hungarian two different terms are used simultaneously, piros and vörös, which differ in their connotations and frequency. Both for ördög and for bor vörös is used. (On the semantic network and distribution of these two color terms see Benczes and Tóth-Czifra 2014). 